\title{
Sulphite: cytochrome $c$ oxidoreductase deficiency in Campylobacter jejuni reduces motility, host cell adherence and invasion
}

\author{
A. Malik Tareen, Javid Iqbal Dasti, Andreas E. Zautner, Uwe Groß \\ and Raimond Lugert \\ University Medical Center Göttingen, Institute for Medical Microbiology, Kreuzbergring 57, \\ 37075 Göttingen, Germany
}

Correspondence

Raimond Lugert

rlugert@gwdg.de

Received 20 September 2010

Revised 15 February 2011

Accepted 2 March 2011

\begin{abstract}
Campylobacter jejuni lacks the enzyme phosphofructokinase and, consequently, is incapable of metabolizing glucose. Instead, the pathogen uses a number of other chemicals to serve as electron donors. Like chemolithotrophic bacteria, C. jejuni is able to respire sulphite in the presence of a sulphite: cytochrome $c$ oxidoreductase (SOR) that is encoded by the genes cj0004c and cj0005c; the former encodes a monohaem cytochrome c oxidoreductase and the latter a molybdopterin oxidoreductase. After screening of a transposon-based mutant library, we identified a mutant with an insertion in gene cj0005c that was strongly reduced in its capacity to infect Caco2 cells. Further characterization of a corresponding non-random knockout mutant together with a complemented mutant and the parental strain showed the cj0005c-deficient mutant to exhibit clearly reduced motility and diminished adherence to host cells. Furthermore, the transcription of genes responsible for the synthesis of, in particular, legionaminic acid was downregulated and the mutant had a reduced capacity to autoagglutinate. In contrast, neither the proliferation of the mutant, nor its intracellular ATP content, was altered compared to the parental strain.
\end{abstract}

\section{INTRODUCTION}

Campylobacter jejuni is the most important cause of bacterial diarrhoea of humans in developing and developed countries (Altekruse et al., 1999; Friedman et al., 2000; Mead et al., 1999). The spectrum of the disease can vary from mild watery diarrhoea to bloody diarrhoea and is characterized by fever and abdominal cramps. In addition, the Guillain-Barré syndrome might emerge as a postinfection complication (Allos, 2001). At the beginning of the infection, $C$. jejuni colonizes the small intestine before moving to the colon, which represents the target organ. C. jejuni is generally considered to be invasive; the level of invasiveness varies depending on the strain (Black et al., 1988; van Vliet \& Ketley, 2001). In contrast to the infection of humans, C. jejuni is only a commensal organism in chickens and other birds, where the pathogen preferentially resides in the caecum, which contains many anaerobic fermentative bacteria (Barnes et al., 1972; Lu et al., 2003). Within those natural habitats, $C$. jejuni is able to metabolize products like free amino and keto acids generated by these accompanying bacterial species or from

Abbreviations: Cp, crossing point; LegAm, legionaminic acid; SOR, sulphite: cytochrome $c$ oxidoreductase.

Three supplementary tables are available with the online version of this paper. the host itself (Lee \& Newell, 2006). Since C. jejuni lacks the glycolytic enzyme phosphofructokinase, glucose cannot be catabolized, and other products serve as electron donors for respiration, for instance succinate, malate, formate, $\mathrm{D}$ lactate, hydrogen and $\mathrm{NAD}(\mathrm{P}) \mathrm{H}$ (Hoffman \& Goodman, 1982; Parkhill et al., 2000; Kelly, 2001; Sellars et al., 2002; Velayudhan \& Kelly, 2002). It has also been shown that sulphite can be exploited as a respiratory electron donor. Like chemolithotrophic bacteria, C. jejuni possesses a sulphite: cytochrome $c$ oxidoreductase (SOR), which is encoded by the genes $c j 0005 c$ and $c j 0004 c$. The $c j 0004 c$ gene encodes a monohaem cytochrome $c$ oxidoreductase, while cj0005c encodes a molybdopterin oxidoreductase (Myers \& Kelly, 2005). The possession of a sulphite oxidation system may be important for the pathogen to survive in foodstuffs that have been treated with sulphite for preservative reasons, or in the human host, since it has been shown that sulphite is also released by neutrophils as part of the host defence (Mitsuhashi et al., 1998). Thus a potential antimicrobial chemical can be used by $C$. jejuni for the generation of electrons, which are subsequently supplied to the respiratory chain of the pathogen and therefore serve for the provision of energy.

In this report we describe that the possession of SOR and the ability to utilize sulphite as an electron donor significantly influences bacterial motility, gene regulation, 
autoagglutination and the invasiveness of $C$. jejuni towards host cells. Bacterial proliferation and intracellular ATP level do not correlate with the presence of this enzyme.

\section{METHODS}

Bacterial strains, media and culture conditions. $C$. jejuni strain B2, isolated from a patient suffering from gastroenteritis, was grown on Columbia agar supplemented with $5 \%$ defibrinated sheep blood at $42{ }^{\circ} \mathrm{C}$ in microaerobic conditions $\left(85 \% \mathrm{~N}_{2}, 10 \% \mathrm{CO}_{2}, 5 \% \mathrm{O}_{2}\right)$ (Schmidt-Ott et al., 2005; Dasti et al., 2007). When needed, kanamycin and chloramphenicol were added at concentrations of $50 \mu \mathrm{g} \mathrm{ml}^{-1}$ and $30 \mu \mathrm{g} \mathrm{ml}^{-1}$, respectively. Growth experiments were carried out at $42{ }^{\circ} \mathrm{C}$ in Mueller-Hinton $(\mathrm{MH})$ broth under microaerobic conditions in the presence or absence of $10 \mathrm{nM}$ $\mathrm{Na}_{2} \mathrm{SO}_{3}$. Cloning experiments were performed in Escherichia coli strain $\mathrm{DH} 5 \alpha$. The E. coli bacteria were grown on Luria Bertani (LB) agar or in LB broth at $37{ }^{\circ} \mathrm{C}$. When necessary, ampicillin $(100 \mu \mathrm{g}$ $\mathrm{ml}^{-1}$ ) was added.

Preparation of competent cells and electroporation. E. coli DH5 $\alpha$ was grown in $500 \mathrm{ml}$ LB broth to an $\mathrm{OD}_{600}$ of $0.35-0.45$. The culture was stored on ice for $10 \mathrm{~min}$ and centrifuged at $4000 \mathrm{~g}$ at $4{ }^{\circ} \mathrm{C}$. After washing the bacteria three times in ice-cold water, the pellet was resuspended in $15 \mathrm{ml} 10 \%(\mathrm{v} / \mathrm{v})$ glycerol and centrifuged again. Finally, the pellet was dissolved in $1 \mathrm{ml} 10 \%(\mathrm{v} / \mathrm{v})$ glycerol and $100 \mu \mathrm{l}$ aliquots were used for electroporation. C. jejuni cells were collected from Columbia agar plates. Then the cells were washed four times in ice-cold washing buffer containing $272 \mathrm{mM}$ sucrose and $15 \%(\mathrm{v} / \mathrm{v})$ glycerol. Finally, the bacteria were resuspended in $400 \mu \mathrm{l}$ washing buffer and aliquots of $100 \mu \mathrm{l}$ were used for electroporation.

For each electroporation of E. coli and C. jejuni, 0.5-3 $\mu$ g plasmid DNA was added to the competent cells and the mixture was transferred into an ice-cold electroporation cuvette. After incubation on ice for $30 \mathrm{~min}$, electroporation was carried out at $2.5 \mathrm{kV}, 25 \mu \mathrm{F}$ and $200 \Omega$ with a BTX Electro Cell manipulator before $500 \mu \mathrm{l}$ SOC medium [2\%(w/v) tryptone, $0.5 \%(\mathrm{w} / \mathrm{v})$ yeast extract, $10 \mathrm{mM} \mathrm{NaCl}$, $2.5 \mathrm{mM} \mathrm{KCl}, 20 \mathrm{mM} \mathrm{MgSO}_{4}, 20 \mathrm{mM}$ glucose] was added. In the case of $C$. jejuni electroporation, the cells were incubated on non-selective Colombia blood agar plates overnight at $37{ }^{\circ} \mathrm{C}$ under microaerobic conditions. Then, the cells were transferred onto plates supplemented with appropriate antibiotics and incubated under microaerobic conditions at $42{ }^{\circ} \mathrm{C}$. Transformed E. coli bacteria were directly plated on selective LB agar and incubated at $37^{\circ} \mathrm{C}$ overnight.

Cultivation of Caco2 cells. Human colon carcinoma Caco2 cells were cultivated in Dulbecco's minimal essential medium (DMEM) supplemented with $10 \%$ fetal bovine serum, $1 \times$ non-essential amino acids, $100 \mathrm{U}$ penicillin $\mathrm{ml}^{-1}$ and $100 \mu \mathrm{g}$ streptomycin $\mathrm{ml}^{-1}$ in a humidified atmosphere of $95 \%$ air and $5 \% \mathrm{CO}_{2}$ at $37{ }^{\circ} \mathrm{C}$.

Invasion and adhesion assays. Bacterial invasion assays were carried out according to Everest et al. (1992). Caco2 cells were grown to approximately $80 \%$ confluence in a six-well plate, then the cells were washed with PBS and inoculated with $400 \mu \mathrm{l} C$. jejuni suspension adjusted to an $\mathrm{OD}_{600}$ of 0.5 , which represents an m.o.i. of 100. In the experiments in which the bacteria were brought into contact with the Caco 2 cells, the six-well plates were centrifuged at $600 \mathrm{~g}$ for $15 \mathrm{~min}$. Two hours post-infection, the bacterial suspension was removed and the cells were washed three times with PBS before further incubation with culture medium supplemented with $100 \mu \mathrm{g}$ gentamicin $\mathrm{ml}^{-1}$. Then the $\mathrm{Caco} 2$ cells were lysed by treatment with $1 \%$ Triton X-100 for $10 \mathrm{~min}$ and the number of viable bacteria was determined by counting c.f.u. on Columbia blood agar plates after incubation for $48 \mathrm{~h}$ at $42{ }^{\circ} \mathrm{C}$ under microaerobic conditions.
For the investigation of bacterial adhesion, $\mathrm{Caco} 2$ cells were incubated with the bacteria for only $30 \mathrm{~min}$. Then the monolayer was washed with PBS, the cells were lysed and the bacteria were plated on Columbia blood agar plates, where the number of recovered bacteria was determined as described above. Every experiment was repeated six times.

ATP assays. To determine the intracellular ATP concentration of bacterial samples, the BacTiter-Glo assay from Promega was used. After growing the bacteria in $\mathrm{MH}$ broth at $42{ }^{\circ} \mathrm{C}$ under microaerobic conditions, cultures were adjusted to an $\mathrm{OD}_{600}$ of 0.5 . Then, serial dilutions of all samples were prepared according to the manufacturer's instructions. Following incubation of the samples at room temperature in a 96-well plate, luminescence was measured together with an ATP standard using a $\operatorname{VICTOR}^{3} \mathrm{~V}$ Multilabel Counter (Perkin Elmer).

Motility assays. One microlitre of an overnight culture that was adjusted to an $\mathrm{OD}_{600}$ of 0.05 was stabbed onto the centre of a $0.4 \%$ $\mathrm{MH}$ agar plate. After incubation of the plate at $42{ }^{\circ} \mathrm{C}$ for $36 \mathrm{~h}$, motility was judged by measuring of the diameter of the halo. Every experiment was carried out four times.

Autoagglutination assays. Autoagglutination assays were carried out as initially described by Misawa \& Blaser (2000). Bacteria grown on plates were suspended in PBS and adjusted to an $\mathrm{OD}_{600}$ of approximately 1.0. A $2 \mathrm{ml}$ sample of each suspension was incubated under microaerobic conditions for $24 \mathrm{~h}$ at $37^{\circ} \mathrm{C}$. Then $1 \mathrm{ml}$ of the bacterial suspension was carefully removed and the $\mathrm{OD}_{600}$ was determined. The parental strain B2, its knockout mutant and the complemented mutant were tested five times each. The data obtained were normalized according to Howard et al. (2009): the $\mathrm{OD}_{600}$ measured after $24 \mathrm{~h}$ was subtracted from the $\mathrm{OD}_{600}$ from the start of the experiment; the values obtained were divided by the initial $\mathrm{OD}_{600}$ and were finally multiplied by 100 in order to give the percentage autoagglutination.

Isolation of nucleic acids. Genomic DNA of C. jejuni was isolated with the QIAamp DNA Mini kit (Qiagen), following the manufacturer's instructions. Plasmid DNA was prepared using the GeneElute Plasmid Miniprep kit (Sigma) according to the manufacturer's protocol. Bacterial RNA from C. jejuni was extracted with the RiboPure Bacter kit (Ambion). DNA contaminations were removed by the treatment with DNase I (Ambion) as recommended by the protocol. Prior to RT-PCR analysis, PCR assays with the isolated bacterial RNA as a template were performed to exclude remaining traces of DNA.

Sequencing of genomic DNA. Genomic DNA sequencing was performed by SeqLab (Göttingen, Germany) using $10 \mu \mathrm{g}$ genomic $C$. jejuni DNA and 10 pmol KanF primer (for all primer sequences, see Supplementary Table S1, available with the online version of this paper).

Insertional knockout of cj0005c. Primers $\mathrm{Cj} 0005 \mathrm{cF}$ and $\mathrm{Cj} 0005 \mathrm{cR}$ (Supplementary Table S1) were used to amplify a 1239 bp DNA fragment corresponding to $c j 0005 c$. The PCR fragment was XbaI digested and inserted into the $\mathrm{XbaI}$-restricted and dephosphorylated plasmid vector pBluescript II KS (Stratagene) to obtain pBcj0005c. For the insertion of a kanamycin-resistance cassette, the aphA-3 gene including a $5^{\prime}$-promoter was amplified from plasmid pSB1699 (Colegio et al., 2001) using the $5^{\prime}$-phosphorylated primers Kan1 and Kan2 (Supplementary Table S1). A PCR containing $10 \mathrm{mM}$ Tris/ $\mathrm{HCl} \mathrm{pH} 8.3,50 \mathrm{mM} \mathrm{KCl}, 1.5 \mathrm{mM} \mathrm{MgCl}_{2}$, all four dNTPs (each $0.2 \mathrm{mM}$ ), $10 \mathrm{pmol}$ primer and $1 \mathrm{U} P f u$ DNA polymerase was carried out to obtain a blunt-ended PCR product. Initial incubation at $95{ }^{\circ} \mathrm{C}$ for $1 \mathrm{~min}$ was followed by 30 cycles at $95{ }^{\circ} \mathrm{C}$ for $30 \mathrm{~s}, 55{ }^{\circ} \mathrm{C}$ for $30 \mathrm{~s}$ 
and $72{ }^{\circ} \mathrm{C}$ for $1 \mathrm{~min}$, with a final incubation at $72{ }^{\circ} \mathrm{C}$ for $10 \mathrm{~min}$. After purification of the PCR product using the QIAquick PCR Purification kit (Qiagen), the kanamycin-resistance cassette was ligated into plasmid pBcj0005c, which was BsaBI-restricted and dephosphorylated with Antarctic Phosphatase (New England Biolabs) to obtain pBcj0005cKan.

Cloning of cj0005c into pRRC. The C. jejuni gene $c j 0005 c$ was amplified by PCR and cloned into C. jejuni expression vector pRRC (Karlyshev \& Wren, 2005). The primers Cj0005cF and Cj0005cR with $\mathrm{Xba \textrm {I }}$ restriction sites are listed in Supplementary Table S1. PCR was carried out using a TRIO-Thermocycler (Biometra) with $10 \mathrm{ng}$ genomic DNA of $C$. jejuni strain B2 as a template. The PCR mixture contained $0.02 \mathrm{U}$ KOD Hot Start DNA Polymerase (Novagene), dNTPs (each $0.2 \mathrm{mM}$ ), $1.5 \mathrm{mM} \mathrm{MgSO}_{4}, 10$ pmol of each primer and $1 \times$ KOD Hot Start buffer. After initial incubation at $95^{\circ} \mathrm{C}$ for $3 \mathrm{~min}$, 40 cycles at $95{ }^{\circ} \mathrm{C}$ for $30 \mathrm{~s}, 55{ }^{\circ} \mathrm{C}$ for $30 \mathrm{~s}$ and $72{ }^{\circ} \mathrm{C}$ for $1 \mathrm{~min}$ were carried out with a final incubation at $72{ }^{\circ} \mathrm{C}$ for $5 \mathrm{~min}$. After PCR, the PCR amplicon and the expression vector pRRC were XbaI digested. Following dephosphorylation of pRRC with Antarctic Phosphatase (New England Biolabs), the restricted PCR fragment was ligated into pRRC using the Quick Ligase kit (New England Biolabs), following the manufacturer's recommendations.

Real-time RT-PCR analysis. Semiquantitative real-time RT-PCR analysis was performed with the LightCycler 1.5 instrument (Roche) and the QuantiFast SYBR Green RT-PCR kit (Qiagen) as described previously (Tareen et al., 2010). In brief, $10 \mathrm{ng}$ samples of RNA isolated from $C$. jejuni B2 wild-type strain, its knockout mutant B2 $\Delta:$ : cj0005c and its complemented knockout mutant were used to compare the transcription levels of the genes summarized in Supplementary Table S1. Amplicons of the $23 S$ gene were used to normalize the RNA pools under investigation based on the crossing points obtained. 23S RT-PCR was carried out as follows. Initial reverse transcription for $20 \mathrm{~min}$ at $50{ }^{\circ} \mathrm{C}$ and denaturation for $5 \mathrm{~min}$ at $95{ }^{\circ} \mathrm{C}$ was followed by 30 cycles of denaturation $\left(95^{\circ} \mathrm{C} ; 10 \mathrm{~s}\right)$, annealing $\left(50{ }^{\circ} \mathrm{C} ; 10 \mathrm{~s}\right)$ and elongation $\left(72{ }^{\circ} \mathrm{C} ; 5 \mathrm{~s}\right)$. After adjustment of the RNA samples with regard to the crossing points of the $23 \mathrm{~S}$ gene, real-time RT-PCR assays were undertaken in order to compare the transcription level of all other investigated genes. Initially, RNA was reverse transcribed for $20 \mathrm{~min}$ at $50{ }^{\circ} \mathrm{C}$ and denatured for $5 \mathrm{~min}$ at $95{ }^{\circ} \mathrm{C}$. Subsequently, 28-35 cycles of denaturation $\left(95^{\circ} \mathrm{C} ; 10 \mathrm{~s}\right)$, annealing $\left(58{ }^{\circ} \mathrm{C} ; 10 \mathrm{~s}\right)$ and elongation $\left(72{ }^{\circ} \mathrm{C}\right.$; $\left.10 \mathrm{~s}\right)$ were executed. The primers used are listed in Supplementary Table S1. Every assay was run in triplicate. Semiquantified transcription levels of the respective genes were calculated as follows: $x=2^{\Delta \mathrm{Cp}}$, where $x$ represents the factor of altered transcription, and $\Delta \mathrm{Cp}$ stands for the difference in crossing points $(\mathrm{Cp} 1-\mathrm{Cp} 2)$ of the sample pools compared.

Statistical analysis. The STATISTICA software (StatSoft) was used to perform Student's $t$-tests. $P$-values $<0.01$ were considered to be significant.

\section{RESULTS}

\section{Identification of cj0005c}

In order to detect genes of $C$. jejuni that are important for the pathogen to invade host cells, we screened a transposonbased mutant library. A total of 660 mutants were examined for their capacity to infect Caco2 cells (Tareen et al., 2010). One of the mutants that showed a clearly reduced infectivity carried a transposon insertion in the gene $c j 0005 c$, which was mapped by direct sequencing of genomic DNA using a primer that binds directly to the $5^{\prime}$ region of the kanamycinresistance cassette (Supplementary Table S1). Gene cj0005c encodes a molybdopterin oxidoreductase, which, together with the adjacent gene $c j 0004 c$ encoding the monohaem cytochrome $c$ oxidoreductase, constitutes a sulphite:cytochrome $c$ oxidoreductase (SOR). In three independent gentamicin protection assays, the percentage of recovered colonies of mutants compared to the parental strain B2 was $24.5,26.5$ and $25 \%$, respectively, with a mean value of $25.34 \%(P<0.001)$.

\section{Construction of a knockout mutant and complementation of the mutant phenotype}

Since it could not be ruled out that the observed invasiondeficient phenotype of $C$. jejuni $\mathrm{B} 2 \Delta c j 0005 \mathrm{c}$ was not due to the transposon insertion into $c j 0005 c$ but rather to genetic rearrangements in the genome, we created a knockout mutant to confirm the above-described observations. After transformation of C. jejuni B2 with pBcj0005cKan, PCR analysis was carried out by using genomic DNA from the obtained C. jejuni clones for the detection of a single band corresponding to the length of cj0005c including the aphA-3 cassette (B2::cj0005c) (Fig. 1b). Subsequent gentamicin protection assays with the generated knockout mutant $\mathrm{B} 2:: c j 0005 c$ confirmed the observed phenotype of $C$. jejuni strain B2 harbouring the transposon within $c j 0005 c$, clearly demonstrating that the inactivation of $c j 0005 c$ was responsible for the invasion-deficient phenotype (Fig. 1c). To restore the phenotype of the parental strain $\mathrm{B} 2$, we introduced the $C$. jejuni expression plasmid pRRC carrying $c j 0005 c$ into $\mathrm{B} 2:: c j 0005 c$ to obtain $\mathrm{B} 2$ :: cj0005c-comp-cj0005c. Because in pRRC the chloramphenicol-resistance cassette and downstream gene cj0005c are flanked by $16 \mathrm{~S}$ and tRNA sequences, a double recombination event leads to the integration of both genes into one of the three $16 \mathrm{~S}$ gene clusters. After verification of the intact $c j 0005 c$ gene by PCR (Fig. 1b), we repeated invasion assays with wild-type strain $\mathrm{B} 2$, both mutants and the complemented knockout mutant. Taking the number of recovered B2 colonies as $100 \%$, the mean values of invasion capacity of $\mathrm{B} 2 \Delta c j 0005 c, \mathrm{~B} 2:: c j 0005 c$ and B2 : : cj0005c-comp-cj0005c were $25 \%, 29.8 \%$ and $85.6 \%$. The altered invasion capacities of both mutants were clearly significant $(P<0.0001)$. In contrast, the $P$-value for the complemented knockout mutant was only 0.16 , indicating no significant difference compared to the parental strain B2 (Fig. 1c). An overview of the genetic arrangement of $c j 0005 c$ in the parental strain, the parental strain with the transposon insertion in $c j 0005 c$, the $c j 0005 c$ knockout mutant and the complemented mutant is shown in Fig. 1(a).

\section{The loss of a functional SOR leads to reduced growth only in the presence of sodium sulphite}

To investigate whether the loss of a functional SOR affects the growth of $C$. jejuni, we compared the growth of the 
(a)

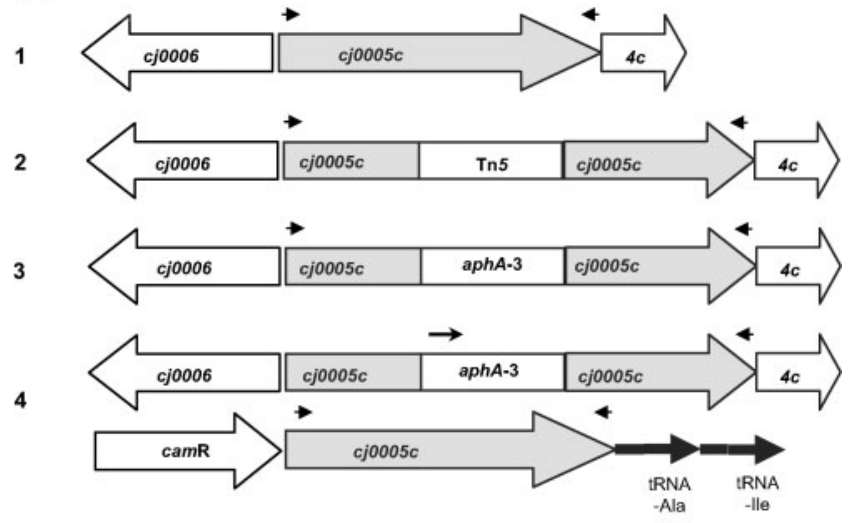

(b)

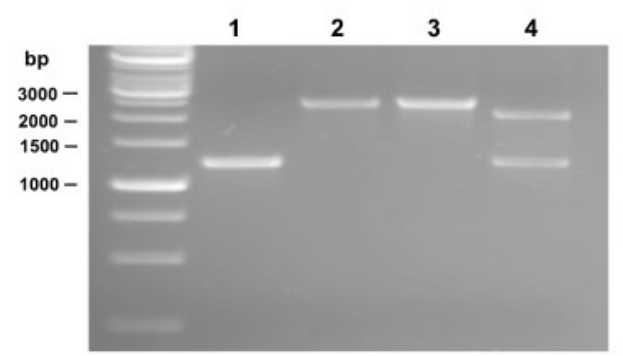

(c)

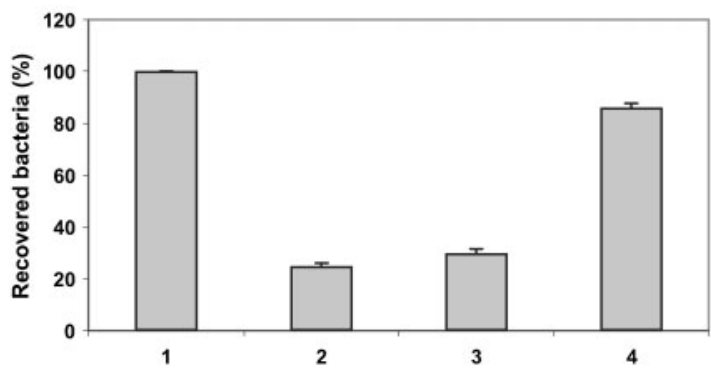

Fig. 1. Key for all panels: 1 , parental strain $B 2 ; 2, B 2$ with transposon insertion in cj0005c (B2Acj0005c); 3, cj0005c knockout mutant (B2::cj0005c); 4, complemented knockout mutant (B2 : : cj0005c-comp-cj0005c). (a) Genome arrangements of the bacterial strains under investigation. Primers $\mathrm{Cj0005cF}$ and $\mathrm{Cj} 0005 \mathrm{cR}$ for the amplification of $\mathrm{cj0005c}$ are indicated as arrowheads. The primer Kan1 that binds to the $5^{\prime}$-end of the kanamycin-resistance cassette is shown as an arrow. (b) Verification of the native gene, the respective mutants and the complemented strain by PCR analysis. The native cj0005c gene from strain B2 has a size of $1239 \mathrm{bp}$ (1). Transposon insertion and insertion of the kanamycin-resistance gene leads to PCR fragments of $2932 \mathrm{bp}$ (2) and $2623 \mathrm{bp}$ (3), respectively. PCR analysis of the complemented knockout mutant using primers $\mathrm{Cj} 0005 \mathrm{cF}$ and $\mathrm{Cj0005cR}$ led only to the amplification of the wildtype gene, probably due to the higher efficiency for the amplification of smaller PCR fragments. To detect the native gene (1239 bp) and the one with the kanamycin-resistance cassette (2234 bp) a PCR analysis with the primer pair $\mathrm{Cj0005cF/}$ $\mathrm{Cj} 0005 \mathrm{cR}$ and an additional third primer (Kan1) binding to the 5 '-end of the aphA-3 gene was carried out. (4). (c) Gentamicin protection assays after the infection of Caco 2 cells. The invasion- deficient phenotype of $\mathrm{B} 2 \Delta \mathrm{cj} 0005 \mathrm{c}$ could be confirmed after knockout of cj0005c (B2::cj0005c). Complementation of the knockout mutant restored the parental phenotype. The data are means \pm SD.

strains in the presence and the absence of sodium sulphite. While in the absence of sodium sulphite no differences in the proliferation of the parental strain B2, the knockout mutant B2::cj0005c and the complemented mutant were observed, the growth of the mutant was clearly reduced as compared to B2 and B2::cj0005c-comp-cj0005c when sodium sulphite was added to the medium at a concentration of $10 \mathrm{mM}$ (Fig. 2).

\section{ATP levels do not differ in the wild-type, mutant and complemented mutant}

Since we could detect no variations in bacterial proliferation of the populations examined in a medium that did not contain the particular substrate for SOR, we asked whether also the ATP level is identical in the parental strain B2 and the $c j 0005 c$-deficient mutant. Upon measuring serial dilutions of the bacteria under investigation with regard to their intracellular ATP content, we could not detect any differences (Fig. 3), demonstrating that the ATP level is not
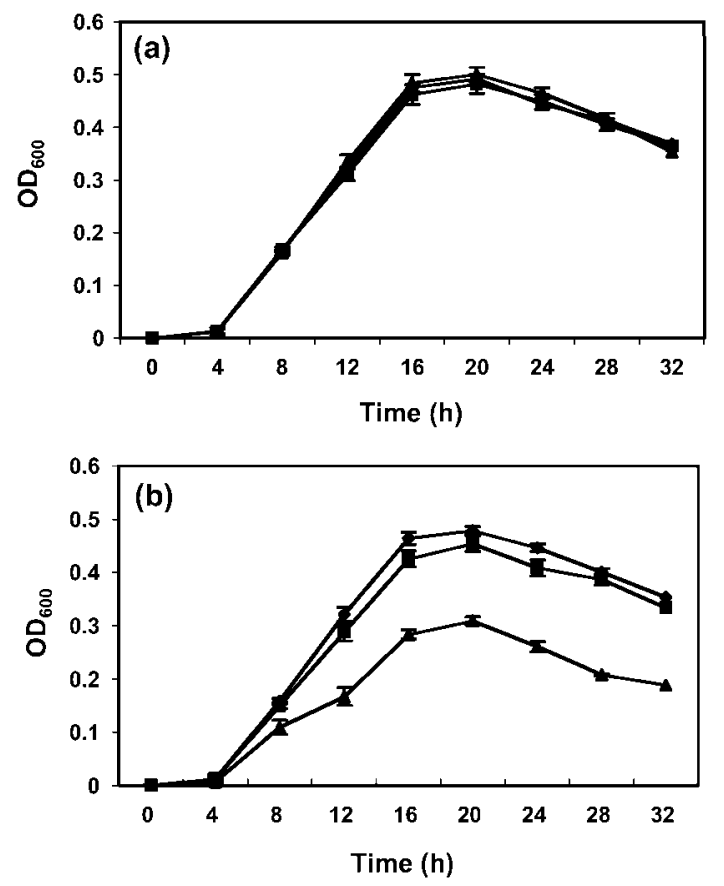

Fig. 2. Growth curves of parental strain B2 $(\boldsymbol{\nabla}), B 2:$ : cj0005c (A) and complemented mutant B2::cj0005c-comp-cj0005c (ש) (a) not supplemented and (b) supplemented with $10 \mathrm{mM} \mathrm{Na}_{2} \mathrm{SO}_{3}$. The growth experiments were repeated three times. Standard deviations are indicated. 
altered even in the absence of an important enzyme, which is an integral part of the energy metabolism.

\section{Reduced motility of $C$. jejuni in the absence of a functional SOR}

We further asked if the motility of the pathogen is diminished by $c j 0005 c$ mutation, since motility is an energy-consuming process. Upon comparing the parental strain B2, its knockout mutant and the complemented knockout mutant, we clearly detected a motility loss of the mutant B2::cj0005c that could be reverted completely to the level of the wild-type strain after introduction of a functional cj0005c gene into the mutant. In four independent motility assays, the mean diameter $( \pm \mathrm{SD})$ of the B2 motility zone was $40 \pm 4.6 \mathrm{~mm}$, while the mutant $\mathrm{B} 2::$ cj0005c was significantly less motile $(18.2 \pm 1.7 \mathrm{~mm}$, $P<0.01)$. Introduction of a functional copy of cj0005c restored the motility to wild-type level again (38.7 \pm $4.1 \mathrm{~mm})$. These findings implied that the reduced infectivity may be primarily due to a lack of motility in the absence of a functional SOR.

\section{Downregulation of flhA, fliR and rpoN transcription in the cj0005c mutant}

To gain an insight into how the lack of a functional SOR is linked to reduced motility, we investigated the regulation of genes which have been shown to be important for the composition of a functional flagellar apparatus (Hendrixson \& DiRita, 2003). We carried out semiquantitive real-time RT-PCR assays to compare the transcription of genes rpoN, flgR, flgS, flhA, flhB, fliP, fliR and maf7 in parental strain $B 2$ and the mutant. While the transcription levels of $f l g S, f l g R$, maf7, $f l h B$ and $f l i P$ were not altered relevantly, we detected a significant downregulation of

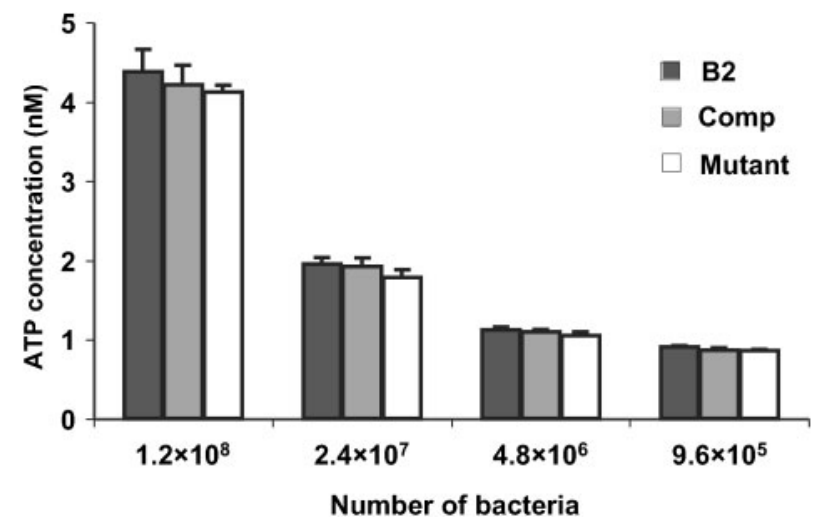

Fig. 3. Intracellular ATP levels of parental strain B2, mutant B2 : :cj0005c and complemented mutant. ATP contents of four serial dilutions of the bacteria under investigation were measured. The results are shown as means \pm SD of data from triplicate wells of a representative experiment. No differences in intracellular ATP content between the bacterial strains could be detected $(P>0.1)$. $r p o N$, fliR and flhA in the mutant strain compared to strain B2; the downregulation of flhA was particularly marked (Table 1). Subsequent testing of the transcription levels of genes significantly downregulated in the mutant revealed only minor differences of the respective crossing points in the parental strain B2 compared to the complemented mutant. The results are shown in Supplementary Table S2.

\section{Reduced motility contributes to decreased invasiveness}

To investigate whether the reduced motility is the only reason for the clearly reduced invasion capacity of the cj0005c-deficient mutant, we performed modified gentamicin protection assays in which motility was mimicked by centrifugation of the bacteria onto the $\mathrm{Caco} 2$ host cells. Taking the number of colonies recovered in the case of wild-type strain B2 as $100 \%$, we detected only $48.6 \%$ of colonies $(P<0.01)$ after centrifugation of the knockout mutant B2 : : cj0005c. The complemented mutant gave $87 \%$ of recovered colonies, which was not significantly different compared to the parental strain (Fig. 4). As compared to the gentamicin protection assays without centrifugation (Fig. 1c) we could clearly observe an increase in recovered mutant colonies from $29.8 \%$ up to $48.6 \%$. Nevertheless, this finding was surprising since one would have expected to shift the mutant invasiveness up to wild-type level after bypassing motility as influencing factor. In contrast, this result indicates that altered motility is one, but not the only factor responsible for the clearly reduced invasiveness of the cj0005c-deficient mutant.

\section{Reduced invasiveness is also caused by diminished adherence}

To characterize the process of invasion in more detail, we investigated whether the adherence capability of the cj0005c-deficient mutant was altered. For this, we carried out adhesion assays of the wild-type isolate $\mathrm{B} 2$, the mutant $\mathrm{B} 2:: c j 0005 c$ and the complemented mutant. After centrifugation of the bacteria onto the host cells, we could clearly determine an adhesion-deficient phenotype of the mutant, which could be restored to wild-type behaviour after complementation. Taking the number of recovered colonies from the parental strain B2 as $100 \%$, the mean value of colonies from the mutant $\mathrm{B} 2:: c j 0005 \mathrm{c}$ was $67 \%$ $( \pm 5.1, P<0.001)$ in contrast to B2::cj0005c-comp-cj0005c with a recovery rate of $94 \%( \pm 2.2)$ (Fig. 4$)$.

\section{The absence of a functional SOR downregulates the transcription of virulence-associated factors}

After the confirmation that the reduced motility in combination with diminished adherence is a reason for the decreased invasiveness of the cj0005c mutant, we examined the transcription level of genes involved in virulence and, in particular, in the adherence of $C$. jejuni to host cells. The investigated genes were groEL and dnaJ, 
Table 1. Transcription levels of genes important for flagellar synthesis in parental strain B2 and mutant strain B2::cj0005c

The crossing point data are means \pm SD. Significantly downregulated genes are in bold.

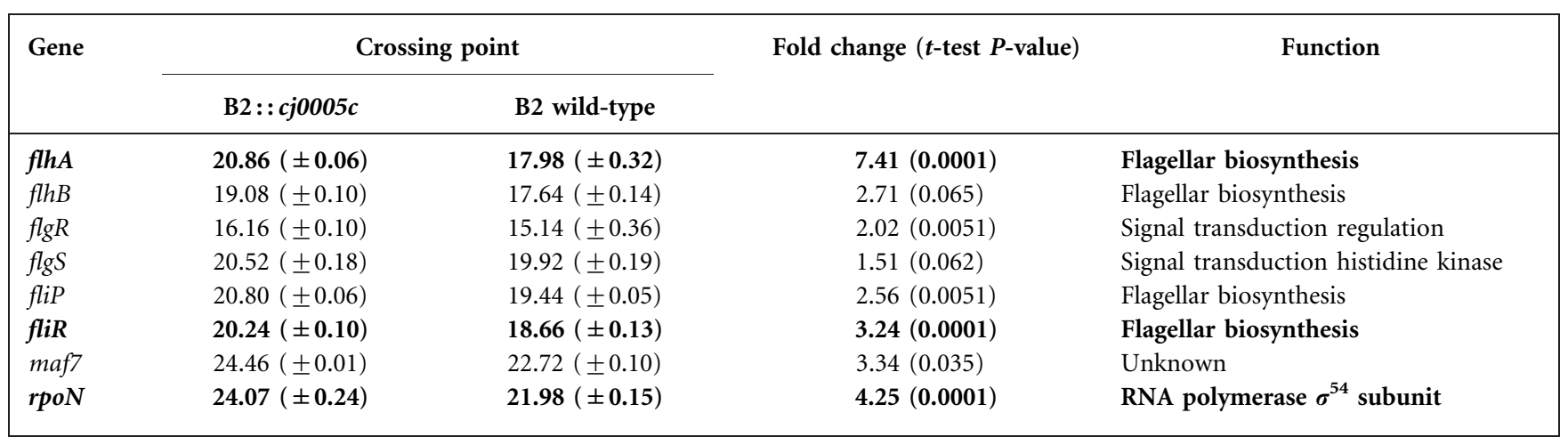

encoding heat-shock proteins (Thies et al., 1999; Konkel et al., 1998), the chemotaxis regulator gene cheY (Yao et al., 1997), the putative chemoreceptor tlp7 (Marchant et al., 2002), genes that encode proteins important for binding and adhesion of C. jejuni, like cadF, pebl, jlpA, porA and flpA (Konkel et al., 1997; Pei \& Blaser 1993; Jin et al., 2001; Flanagan et al., 2009; Konkel et al., 2010) and genes known to be responsible for $O$-linked glycosylation of the flagella by the synthesis of pseudaminic acid and legionaminic acid (LegAm) (Logan et al., 2008). In addition, we compared the transcription level of Campylobacter invasion antigen gene ciaB (Rivera-Amill et al., 2001; Konkel et al., 2004), the cytolethal distending toxin subunit B gene $c d t B$ (Pickett \& Whitehouse, 1999) and flaA (Guerry et al., 1991) in the wild-type strain B2 and its mutant. Semiquantitative

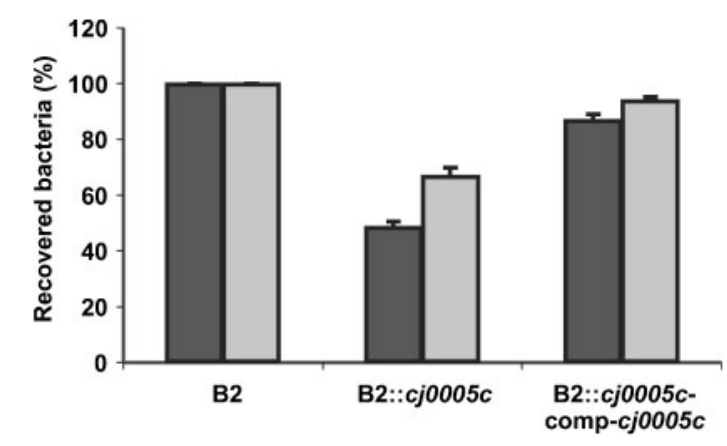

Fig. 4. Invasion and adhesion assays with wild-type strain B2, knockout mutant $\mathrm{B} 2:: \mathrm{cj} 0005 \mathrm{c}$ and complemented mutant B2 : : cj0005c-comp-cj0005c. Dark grey bars: gentamicin protection invasion assays after centrifugation of the bacteria onto Caco2 cells. Taking the number of $C$. jejuni strain B2 colonies recovered as $100 \%$, the mean percentage of knockout mutant B : : cj0005c colonies was $48.6 \%$. Complementation of the mutant partially restored the parental phenotype ( $87 \%$ recovery). See text for details. Light grey bars: adhesion assays after centrifuging of the bacteria onto Caco2 cells. The percentage of adhered colonies of the mutant B2::cj0005c was only $67 \%$ compared to wild-type isolate B2. real-time RT-PCR assays detected a statistically significant 5.16-fold downregulation of dnaJ transcription in the mutant compared to the transcription level of this gene in wild-type strain B2 $(P<0.001)$. In addition, the transcription of $p t m B, p t m C$ and $p t m E$, which contribute to a set of genes responsible for the synthesis of LegAm were significantly downregulated by a factor of 4.31-7.67 $(P<0.001)$ as outlined in Table 2. The transcription of other genes, including genes of LegAm metabolism, were also downregulated significantly, but, to a lesser extent ( $p t m A, p t m F, p t m G)$. Other virulence factors investigated, e.g. for the biosynthesis of pseudaminic acid, were not controlled or only regulated by a factor of uncertain biological significance. The results are summarized in Supplementary Table S3.

Further, we tested in the cj0005c-complemented mutant the transcription of the genes judged to be significantly downregulated in the cj0005c-mutant. The results showed crossing points on the same transcription level as for the parental strain (Supplementary Table S2).

\section{Autoagglutination deficiency of mutant B2 : : cj0005c}

Howard et al. (2009) demonstrated that C. jejuni mutants lacking gene cj1324 or genes cj1321-1325/6, known to be responsible for the synthesis of LegAm, had significantly lower levels of autoagglutination compared to the parental strain. By comparing the transcription profile of the mutant B2::cj0005c with that of the wild-type strain B2 we were able to detect a significant downregulation of transcription of $p t m G$ (cj1324) as well as that of other genes involved in the synthesis of LegAm as described above. We therefore tested whether the competence of mutant B2: : cj0005c is also affected in regard to autoagglutination. We found that the autoagglutination capability of the mutant is indeed decreased. Whereas the percentage autoagglutination of wild-type strain B2 and the complemented mutant was $74.24 \%( \pm 0.25)$ and $73.65 \%$ $( \pm 0.22)$, respectively, the mutant $\mathrm{B} 2::$ cj0005c had an 
Table 2. Transcription level of genes responsible for the synthesis of LegAm and the gene encoding heat-shock protein DnaJ in C. jejuni wild-type strain B2 and mutant B2::cj0005c

The crossing point data are means \pm SD. Strongly downregulated genes are in bold. See text for details.

\begin{tabular}{|c|c|c|c|c|}
\hline \multirow[t]{2}{*}{ Gene } & \multicolumn{2}{|c|}{ Crossing point } & \multirow{2}{*}{$\begin{array}{c}\text { Fold change }(t \text {-test } \\
P \text {-value })\end{array}$} & \multirow[t]{2}{*}{ Function } \\
\hline & $\mathrm{B} 2:: c j 0005 c$ & B2 wild-type & & \\
\hline dnaJ $(c j 1260 c)$ & $23.34( \pm 0.09)$ & $20.97( \pm 0.05)$ & $5.16(0.0001)$ & Heat shock response \\
\hline ptmA (cj1332) & $22.29( \pm 0.55)$ & $20.79( \pm 0.17)$ & $2.82(0.0001)$ & LegAm synthesis \\
\hline ptmB $(c j 1331)$ & $19.40( \pm 0.77)$ & $16.83( \pm 0.01)$ & $5.93(0.0001)$ & LegAm synthesis \\
\hline ptmC (cj1327) & $18.31( \pm 0.47)$ & $16.20( \pm 1.19)$ & $4.31(0.0001)$ & LegAm synthesis \\
\hline ptmD (cj1328) & $20.53( \pm 0.59)$ & $20.16( \pm 0.72)$ & $1.29(0.39)$ & LegAm synthesis \\
\hline ptmE (cj1329) & $18.11( \pm 0.06)$ & $15.17( \pm 0.38)$ & $7.67(0.0001)$ & LegAm synthesis \\
\hline ptmF (cj1330) & $19.74( \pm 0.50)$ & $18.30( \pm 0.89)$ & $2.71(0.0001)$ & LegAm synthesis \\
\hline$p t m G(c j 1324)$ & $19.16( \pm 0.25)$ & $17.64( \pm 0.15)$ & $2.86(0.0001)$ & LegAm synthesis \\
\hline$p t m H(c j 1325 / 6)$ & $20.17( \pm 0.26)$ & $18.82( \pm 0.12)$ & $2.54(0.017)$ & LegAM synthesis \\
\hline
\end{tabular}

autoagglutination level of only $37.02 \%( \pm 0.61)$, which was significantly reduced compared to B2 $(P<0.001)$.

\section{DISCUSSION}

C. jejuni possesses a highly branched and complex respiratory chain that allows the pathogen to use a variety of electron donors such as D-lactate, hydrogen, formate, succinate, malate, $\mathrm{NAD}(\mathrm{P}) \mathrm{H}$ and sulphite (Kelly, 2001; Sellars et al., 2002; Myers \& Kelly, 2005; Weerakoon et al., 2009). The enzyme responsible for the oxidation of sulphite to sulphate and the release of electrons applied into the respiratory chain is a molybdenum-containing sulphite:cytochrome $c$ oxidoreductase (SOR) which is composed of $\mathrm{Cj} 0005 \mathrm{c}$ and $\mathrm{Cj0004c}$ and has homology to the corresponding enzyme of Starkeya novella. The two subunits were shown to constitute a functional SOR which respires sulphite in the presence of oxygen. The SORgenerated electrons, in turn, enter the respiratory chain after the $b c_{1}$ complex at the level of cytochrome $c$ (Kappler et al., 2000; Kappler \& Dahl, 2001; Myers \& Kelly, 2005).

In this report, we describe a $C$. jejuni cj0005c mutant that has strongly reduced motility and, therefore, a reduced capacity to invade host cells. In contrast to other motilityreduced mutants, no gene of the flagellar apparatus itself is affected, but SOR, an enzyme mediating the delivery of electrons into the respiratory chain of the pathogen. Motility was shown to be important for $C$. jejuni to colonize and invade the respective host. Several studies in recent years have described the impact of genes and proteins of the flagellar apparatus on host colonization or the invasion of host cells in particular. One of the first publications, by Morooka et al. (1985), described that nonmotile C. jejuni strains were cleared from the intestinal tract of suckling mice, whereas a wild-type strain colonized the lower portion of the intestine, the caecum and the colon. Furthermore, the colonization of rabbits was shown to depend on the flagellum, since a non-flagellated mutant was unable to survive inside the rabbit intestine (Pavlovskis et al., 1991). In addition to the studies mentioned above, the chicken intestinal tract was shown to be colonized only by fully motile strains of C. jejuni, which demonstrated that an intact flagellum is an important colonization factor (Nachamkin et al., 1993). Homologous recombination techniques were successfully applied to generate mutants that subsequently were investigated in cell culture experiments for their ability to invade host cells. Thereby, the importance of motility for the invasiveness of $C$. jejuni could be confirmed (Wassenaar et al., 1991; Yao et al., 1994; Szymanski et al., 1995; Konkel et al., 2004). Meanwhile, many genes and proteins have been characterized which contribute to an intact flagellum in order to guarantee motility and, therefore, infectivity (Hendrixson, 2008). In order to find a link between the absence of a functional SOR on the one hand, and the reduced motility of the mutant on the other hand, we investigated the transcription levels of genes known to be important for flagellar composition. We detected a significant downregulation of genes $f l i R$ and $f l h A$, which encode proteins shown to be essential for the expression of FlgD and FlgE2, and of rpoN, encoding the RNA polymerase $\sigma^{54}$ subunit of the pathogen, known to be responsible for the expression of the flgDE2 locus as well as for the expression of minor flagellin FlaB (Hendrixson et al., 2001; Hendrixson \& DiRita, 2003). These results indicate that the motility of the SOR mutant is negatively affected, although we have no data on protein level regarding the proteins whose expression is controlled by the gene products of fliR, flhA or $\operatorname{ropN}$.

The finding that the loss of a gene which is responsible for the supply of electrons into the respiratory chain leads to reduced motility raised the question whether the reduced motility is linked to the impaired energy metabolism. However, when we compared the intracellular ATP level of B2::cj0005c with the wild-type strain and the cj0005ccomplemented mutant, we detected no differences. In addition, the cell proliferation of the mutant was not 
negatively affected when the bacteria were grown in $\mathrm{MH}$ broth. Only in the presence of sodium sulphite was the mutant's growth clearly impaired, probably due to the reduced ability of the mutant to clear this potentially antimicrobial chemical.

Surprisingly, when we eliminated motility as a virulenceassociated factor by centrifuging the parental strain B2 and its mutant directly onto the host cells, we were able to shift the infectivity of the mutant from $29.8 \%$ to only $48.6 \%$, indicating that not only reduced motility is responsible for the mutant's impaired invasiveness compared to the wildtype strain. To obtain a more detailed insight into the mechanism of host cell invasion, adhesion assays were carried out. These experiments detected a reduced capacity of mutant B2::cj0005c compared to the wild-type strain in this initial host-pathogen interaction. Since many $C$. jejuni genes which contribute to the virulence of the pathogen and to the process of adhesion in particular have been described, we investigated whether the loss of a functional SOR has an impact on the transcription level of these genes. C. jejuni genes involved in stress responses, motility or chemotaxis were shown to be essential for the invasion of host cells. In addition, genes important for binding and adhesion were examined. Associated with the gene encoding the heat-shock protein DnaJ, the transcription of several genes responsible for the synthesis of legionaminic acid (LegAm) were shown to be significantly downregulated in the mutant $\mathrm{B} 2::$ cj0005c.

The acetoamidino form of LegAm together with pseudaminic acid are two distinct nine-carbon sugars, which are $O$-linked to flagellin in most C. jejuni strains (Logan et al., 2008). Altogether eight genes have been identified which are required for the synthesis of LegAm. These so-called post-translational modification $(p t m)$ genes are cj1324 (ptmG), cj1325/6 (ptmH), cj1327-cj1330 (ptmC, ptmD, ptmE, ptmF), cj1331 (ptmB) and cj1332 (ptmA) (McNally et al., 2007). Although knowledge of the importance of $O$ glycosylation by LegAm is limited, recent investigations by Howard et al. (2009) gave a first insight into its biological relevance. A C. jejuni cj1324 mutant showed full motility, but less hydrophobicity and impaired autoagglutination and biofilm formation due to the absence of two LegAm glycan modifications. Furthermore, this mutant had a reduced capacity to colonize chickens. When we investigated the transcription level of the LegAm genes in the cj0005c mutant, we detected only a 2.86-fold downregulation of cj1324 (ptmG). However, we also could clearly show reduced autoagglutination but no alterations in biofilm formation (data not shown). In contrast to Howard et al. (2009), we did not investigate the genes cj1321-cj1323, but we examined all genes required for LegAm synthesis with regard to altered transcription. This revealed transcription of $p t m B$ (cj1331), ptmC (cj1327) and ptmE (cj1329) to be strongly downregulated in the cj0005c mutant, whereas the transcription of genes ptmA (cj1332), $p t m F$ (cj1330) and ptmG (cj1324) was significantly downregulated but to a lesser extent. However, we have no data regarding the corresponding proteins, and the data obtained so far and the resulting conclusions refer exclusively to our findings of reduced transcription. Since we have not performed transcription analysis covering the whole genome of the pathogen, alterations in the transcription level of other genes are likely. But the results obtained so far might indicate a correlation between the mutant's altered biological properties (in particular diminished adhesion and autoagglutination) and the downregulation of genes responsible for LegAm synthesis.

In this study, we have characterized an invasion-deficient mutant, which owes its particular phenotype not to a mutation in a gene directly responsible in the process of motility, adherence or infection, but to a mutation within a gene involved in the energy metabolism of the pathogen. Despite this, we demonstrated an influence on events that are necessary for host cell invasion. It cannot be excluded that the reduced motility and the downregulation of virulence-associated genes are connected to the attempt of the pathogen to maintain the intracellular ATP level even in the absence of an enzyme which provides electrons for the respiratory chain. Other $C$. jejuni mutants with restricted energy metabolism should be examined in the future in order to investigate if a constant ATP level accompanying reduced biological functions and downregulated transcription is a more general phenomenon in C. jejuni.

\section{ACKNOWLEDGEMENTS}

This work was supported by the Deutsche Forschungsgemeinschaft (GR 906/13-1). A. M. T. was supported by a grant from the German Academic Exchange Service (DAAD). We thank the CASVAB, University of Balochistan, Pakistan and the Higher Education Commission, Pakistan.

\section{REFERENCES}

Allos, B. M. (2001). Campylobacter jejuni infections: update on emerging issues and trends. Clin Infect Dis 32, 1201-1206.

Altekruse, S. F., Stern, N. J., Fields, P. I. \& Swerdlow, D. L. (1999). Campylobacter jejuni - an emerging foodborne pathogen. Emerg Infect Dis 5, 28-35.

Barnes, E. M., Mead, G. C., Barnum, D. A. \& Harry, E. G. (1972). The intestinal flora of the chicken in the period 2 to 6 weeks of age, with particular reference to the anaerobic bacteria. Br Poult Sci 13, 311326.

Black, R. E., Levine, M. M., Clements, M. L., Hughes, T. P. \& Blaser, M. J. (1988). Experimental Campylobacter jejuni infection in humans. $J$ Infect Dis 157, 472-479.

Colegio, O. R., Griffin, T. J., IV, Grindley, N. D. F. \& Galán, J. E. (2001). In vitro transposition system for efficient generation of random mutants of Campylobacter jejuni. J Bacteriol 183, 2384-2388.

Dasti, J. I., Gross, U., Pohl, S., Lugert, R., Weig, M. \& Schmidt-Ott, R. (2007). Role of the plasmid-encoded $\operatorname{tet}(O)$ gene in tetracyclineresistant clinical isolates of Campylobacter jejuni and Campylobacter coli. J Med Microbiol 56, 833-837.

Everest, P. H., Goossens, H., Butzler, J. P., Lloyd, D., Knutton, S., Ketley, J. M. \& Williams, P. H. (1992). Differentiated Caco-2 cells 
as a model for enteric invasion by Campylobacter jejuni and C. coli. J Med Microbiol 37, 319-325.

Flanagan, R. C., Neal-McKinney, J. M., Dhillon, A. S., Miller, W. G. \& Konkel, M. E. (2009). Examination of Campylobacter jejuni putative adhesins leads to the identification of a new protein, designated FlpA, required for chicken colonization. Infect Immun 77, 2399-2407.

Friedman, C. R., Neiman, J., Wegener, H. C. \& Tauxe, R. V. (2000). Epidemiology of Campylobacter jejuni in the United States and other industrialized nations. In Campylobacter, 2nd edn, pp. 121-138. Edited by I. Nachamkin \& M. J. Blaser. Washington, DC: American Society for Microbiology.

Guerry, P., Alm, R. A., Power, M. E., Logan, S. M. \& Trust, T. J. (1991). Role of two flagellin genes in Campylobacter motility. J Bacteriol 173, 4757-4764.

Hendrixson, D. R. (2008). Regulation of flagellar gene expression and regulation. In Campylobacter, 3rd edn, pp. 545-558. Edited by I. Nachamkin, C. M. Szymanski \& M. J. Blaser. Washington, DC: American Society for Microbiology.

Hendrixson, D. R. \& DiRita, V. J. (2003). Transcription of $\sigma^{54}$ dependent but not $\sigma^{28}$-dependent flagellar genes in Campylobacter jejuni is associated with formation of the flagellar secretory apparatus. Mol Microbiol 50, 687-702.

Hendrixson, D. R., Akerley, B. J. \& DiRita, V. J. (2001). Transposon mutagenesis of Campylobacter jejuni identifies a bipartite energy taxis system required for motility. Mol Microbiol 40, 214-224.

Hoffman, P. S. \& Goodman, T. G. (1982). Respiratory physiology and energy conservation efficiency of Campylobacter jejuni. J Bacteriol 150, 319-326.

Howard, S. L., Jagannathan, A., Soo, E. C., Hui, J. P. M., Aubry, A. J., Ahmed, I., Karlyshev, A., Kelly, J. F., Jones, M. A. \& other authors (2009). Campylobacter jejuni glycosylation island important in cell charge, legionaminic acid biosynthesis, and colonization of chickens. Infect Immun 77, 2544-2556.

Jin, S., Joe, A., Lynett, J., Hani, E. K., Sherman, P. \& Chan, V. L. (2001). JlpA, a novel surface-exposed lipoprotein specific to Campylobacter jejuni, mediates adherence to host epithelial cells. Mol Microbiol 39, $1225-1236$.

Kappler, U. \& Dahl, C. (2001). Enzymology and molecular biology of prokaryotic sulfite oxidation. FEMS Microbiol Lett 203, 1-9.

Kappler, U., Bennett, B., Rethmeier, J., Schwarz, G., Deutzmann, R., McEwan, A. G. \& Dahl, C. (2000). Sulfite:cytochrome $c$ oxidoreductase from Thiobacillus novellus. Purification, characterization, and molecular biology of a heterodimeric member of the sulfite oxidase family. J Biol Chem 275, 13202-13212.

Karlyshev, A. V. \& Wren, B. W. (2005). Development and application of an insertional system for gene delivery and expression in Campylobacter jejuni. Appl Environ Microbiol 71, 4004-4013.

Kelly, D. J. (2001). The physiology and metabolism of Campylobacter jejuni and Helicobacter pylori. J Appl Microbiol 175, 102-111.

Konkel, M. E., Garvis, S. G., Tipton, S. L., Anderson, D. E., Jr \& Cieplak, W., Jr (1997). Identification and molecular cloning of a gene encoding a fibronectin-binding protein (CadF) from Campylobacter jejuni. Mol Microbiol 24, 953-963.

Konkel, M. E., Kim, B. J., Klena, J. D., Young, C. R. \& Ziprin, R. (1998). Characterization of the thermal stress response of Campylobacter jejuni. Infect Immun 66, 3666-3672.

Konkel, M. E., Klena, J. D., Rivera-Amill, V., Monteville, M. R., Biswas, D., Raphael, B. \& Mickelson, J. (2004). Secretion of virulence proteins from Campylobacter jejuni is dependent on a functional flagellar export apparatus. J Bacteriol 186, 3296-3303.
Konkel, M. E., Larson, C. L. \& Flanagan, R. C. (2010). Campylobacter jejuni FlpA binds fibronectin and is required for maximal host cell adherence. J Bacteriol 192, 68-76.

Lee, M. D. \& Newell, D. G. (2006). Campylobacter in poultry: filling an ecological niche. Avian Dis 50, 1-9.

Logan, S. M., Schoenhofen, I. C. \& Guerry, P. (2008). O-linked flagellar glycosylation in Campylobacter. In Campylobacter, 3rd edn, pp. 471-481. Edited by I. Nachamkin, C. M. Scymanski \& M. J. Blaser. Washington, DC: American Society for Microbiology.

Lu, J., Idris, U., Harmon, B., Hofacre, C., Maurer, J. J. \& Lee, M. D. (2003). Diversity and succession of the intestinal bacterial community of the maturing broiler chicken. Appl Environ Microbiol 69, 68166824 .

Marchant, J., Wren, B. \& Ketley, J. (2002). Exploiting genome sequence: predictions for mechanisms of Campylobacter chemotaxis. Trends Microbiol 10, 155-159.

McNally, D. J., Aubry, A. J., Hui, J. P., Khieu, N. H., Whitfield, D., Ewing, C. P., Guerry, P., Brisson, J. R., Logan, S. M. \& Soo, E. C. (2007). Targeted metabolomics analysis of Campylobacter coli VC167 reveals legionaminic acid derivatives as novel flagellar glycans. J Biol Chem 282, 14463-14475.

Mead, P. S., Slutsker, L., Dietz, V., McCaig, L. F., Bresee, J. S., Shapiro, C., Griffin, P. M. \& Tauxe, R. V. (1999). Food-related illness and death in the United States. Emerg Infect Dis 5, 607-625.

Misawa, N. \& Blaser, M. J. (2000). Detection and characterization of autoagglutination activity by Campylobacter jejuni. Infect Immun 68, 6168-6175.

Mitsuhashi, H., Nojima, Y., Tanaka, T., Ueki, K., Maezawa, A., Yano, S. \& Naruse, T. (1998). Sulfite is released by human neutrophils in response to stimulation with lipopolysaccharide. J Leukoc Biol 64, 595599.

Morooka, T., Umeda, A. \& Amako, K. (1985). Motility as an intestinal colonization factor for Campylobacter jejuni. J Gen Microbiol 131, 1973-1980.

Myers, J. D. \& Kelly, D. J. (2005). A sulphite respiration system in the chemoheterotrophic human pathogen Campylobacter jejuni. Microbiology 151, 233-242.

Nachamkin, I., Yang, X. H. \& Stern, N. J. (1993). Role of Campylobacter jejuni flagella as colonization factors for three-dayold chicks: analysis with flagellar mutants. Appl Environ Microbiol 59, 1269-1273.

Parkhill, J., Wren, B. W., Mungall, K., Ketley, J. M., Churcher, C., Basham, D., Chillingworth, T., Davies, R. M., Feltwell, T. \& other authors (2000). The genome sequence of the food-borne pathogen Campylobacter jejuni reveals hypervariable sequences. Nature 403, 665-668.

Pavlovskis, O. R., Rollins, D. M., Haberberger, R. L., Jr, Green, A. E., Habash, L., Strocko, S. \& Walker, R. I. (1991). Significance of flagella in colonization resistance of rabbits immunized with Campylobacter spp. Infect Immun 59, 2259-2264.

Pei, Z. M. \& Blaser, M. J. (1993). PEB1, the major cell-binding factor of Campylobacter jejuni, is a homolog of the binding component in Gram-negative nutrient transport systems. J Biol Chem 268, 1871718725.

Pickett, C. L. \& Whitehouse, C. A. (1999). The cytolethal distending toxin family. Trends Microbiol 7, 292-297.

Rivera-Amill, V., Kim, B. J., Seshu, J. \& Konkel, M. E. (2001). Secretion of the virulence-associated Campylobacter invasion antigens from Campylobacter jejuni requires a stimulatory signal. J Infect Dis 183, 1607-1616. 
Schmidt-Ott, R., Pohl, S., Burghard, S., Weig, M. \& Gross, U. (2005). Identification and characterization of a major subgroup of conjugative Campylobacter jejuni plasmids. J Infect 50, 12-21.

Sellars, M. J., Hall, S. J. \& Kelly, D. J. (2002). Growth of Campylobacter jejuni supported by respiration of fumarate, nitrate, nitrite, trimethylamine- $N$-oxide, or dimethyl sulfoxide requires oxygen. $J$ Bacteriol 184, 4187-4196.

Szymanski, C. M., King, M., Haardt, M. \& Armstrong, G. D. (1995). Campylobacter jejuni motility and invasion of Caco-2 cells. Infect Immun 63, 4295-4300.

Tareen, A. M., Dasti, J. I., Zautner, A. E., Gross, U. \& Lugert, R. (2010). Campylobacter jejuni proteins Cj0952c and Cj0951c affect chemotactic behaviour towards formic acid and are important for invasion of host cells. Microbiology 156, 3123-3135.

Thies, F. L., Weishaupt, A., Karch, H., Hartung, H. P. \& Giegerich, G. (1999). Cloning, sequencing and molecular analysis of the Campylobacter jejuni groESL bicistronic operon. Microbiology 145, 89-98.

van Vliet, A. H. \& Ketley, J. M. (2001). Pathogenesis of enteric Campylobacter infection. Symp Ser Soc Appl Microbiol 30, 45S-56S.
Velayudhan, J. \& Kelly, D. J. (2002). Analysis of gluconeogenic and anaplerotic enzymes in Campylobacter jejuni: an essential role for phosphoenolpyruvate carboxykinase. Microbiology 148, 685694.

Wassenaar, T. M., Bleumink-Pluym, N. M. \& van der Zeijst, B. A. (1991). Inactivation of Campylobacter jejuni flagellin genes by homologous recombination demonstrates that flaA but not flaB is required for invasion. EMBO J 10, 2055-2061.

Weerakoon, D. R., Borden, N. J., Goodson, C. M., Grimes, J. \& Olson, J. W. (2009). The role of respiratory donor enzymes in Campylobacter jejuni host colonization and physiology. Microb Pathog 47, 8-15.

Yao, R., Burr, D. H., Doig, P., Trust, T. J., Niu, H. \& Guerry, P. (1994). Isolation of motile and non-motile insertional mutants of Campylobacter jejuni: the role of motility in adherence and invasion of eukaryotic cells. Mol Microbiol 14, 883-893.

Yao, R., Burr, D. H. \& Guerry, P. (1997). CheY-mediated modulation of Campylobacter jejuni virulence. Mol Microbiol 23, 1021-1031.

Edited by: A. H. M. van Vliet 